\title{
Tonal fusion of consonant musical intervals: The oomph in Stumpf
}

\author{
LUCINDA A. DEWITT and ROBERT G. CROWDER \\ Yale University, New Haven, Connecticut
}

\begin{abstract}
Three experiments investigated Stumpf's fusion principle of tonal consonance. In Experiment 1, subjects listened to single tones or to tone pairs that represented 12 musical intervals and indicated whether they thought one or two tones had sounded. The fusion principle would be supported by an increase in reaction times or errors in response to conventionally consonant intervals as opposed to dissonant ones. A significant main effect of interval size was found in the error data of Experiment 1, with the most errors produced by intervals of an octave (12 semitones). Experiment 2 compared a smaller set of intervals in distinguishing two-from three-tone combinations. A significant main effect of interval size was found in both the response time and error data in a direction consistent with the fusion principle. Experiment 3 investigated an explanation of the fusion principle based on the harmonic series. In three-tone combinations, more fusion of the higher pair of tones was observed when the lower pair formed an octave or perfect fifth than when the lower pair formed a tritone. Fusion may represent a tendency for people to interpret pitch combinations that could represent harmonics resulting from a single fundamental as timbres rather than as chords.
\end{abstract}

The perceived consonance and dissonance of pairs of tones has been of musical interest for over 2,500 years. Since Pythagoras's time, many theories have been proposed to explain why some combinations of tones sound more pleasant (consonant) and others more unpleasant (dissonant). These explanations of consonance and dissonance fall into two general categories: (1) "rational" theories with an acoustic basis in "natural law," or simple integer ratios, and (2) "empirical" theories based on context and learning (or brainwashing) (Cazden, 1980; see also Terhardt, 1984).

Today the accepted view of consonance and dissonance includes aspects of both approaches. Two types of consonance are distinguished, tonal consonance and musical consonance. Tonal consonance refers to "the particular sensory experience associated to isolated tone pairs with simple frequency ratios" (Plomp \& Levelt, 1965, p. 548). Musical consonance, on the other hand, refers to the functional relationships brought about by a "sonorous event ... expected to resolve" (dissonance) and "the moment to which it ultimately resolves"' (consonance) (Cazden, 1980 , p. 157). These functional definitions of musical

\footnotetext{
Preparation of this article was supported under a National Science Foundation graduate fellowship to Lucinda DeWitt and by National Science Foundation Grant BNS 82-19661 to Robert Crowder. The authors appreciate the use of facilities at Haskins Laboratories (NICHD Contract N01 HD52910) and the assistance of Bruno Repp in stimulus analysis. A preliminary version of Experiment 1 was conducted by the second author with Richard E. Pollinger in December 1957. This was a project for an introductory psychology course at the University of Michigan. The late Professor Carl Brown suggested the idea behind the study and the methodology for testing it. The authors are indebted to him most of all. Requests for reprints should be sent to either author at the Department of Psychology, Yale University, Box 11A, Yale Station, New Haven, CT 06520.
}

consonance and dissonance depend on the existence of a "framework for the normative expectations" (Cazden, 1980 , p. 158) such as that defined within a certain stylistic period.

Of the two types of consonance, tonal consonance has been the more thoroughly investigated experimentally and is the subject of this report. In general, investigations of tonal consonance require listeners to judge or rate pairs of tones as to their consonance-dissonance, smoothnessroughness, or pleasantness-unpleasantness. Although the use of verbal labels is a perfectly valid way of measuring consonance and dissonance, converging evidence from a nonverbal, more objective measure would certainly add to our understanding of this phenomenon. The idea for our choice of a nonverbal measure came from Stumpf's principle of tonal fusion (as described in Apel, 1972, p. 201, Boring, 1942, and Roberts, 1983).

Stumpf's doctrine of tonal fusion holds that "tones tend to fuse, to interpenetrate each other, with the result that the total perception becomes something different from the mere concurrence of its components (as the Gestalt psychologists also said later), and that different combinations vary in their degree of fusion"' (Boring, 1942, p. 360). Stumpf conducted an experiment that provided evidence in support of his fusion principle. Untrained subjects listened to simultaneously presented musical intervals and reported their introspections (single tone or two different tones) of the pairs. The measure of degree of perceived consonance was the percentage of subjects falsely judging a pair of tones as "single tone." Figure 1 illustrates the results (data from Apel, 1972).

Even setting aside our skepticism about experimental procedures and demand characteristics that could compromise these data, we might wonder whether reaction 


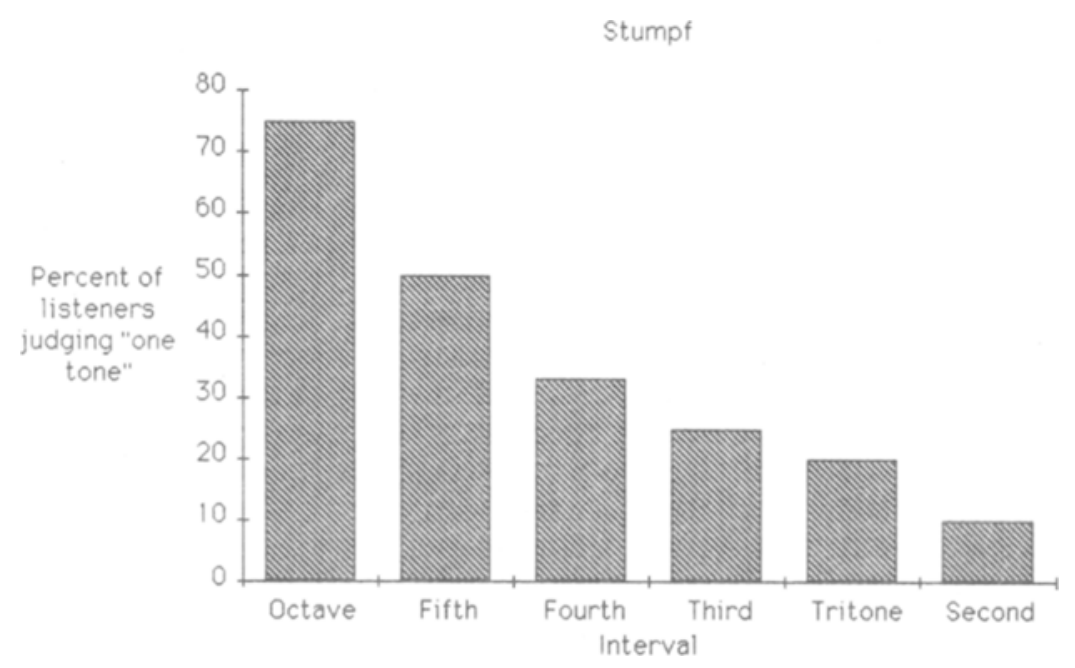

Figure 1. Percentage of listeners judging "one tone" to a tone pair as a function of the musical interval of the pair (experiment by Stumpf, data from Apel, 1972).

time would not be a better measure. A longer response time to indicate "two tones" when the pair of tones forms a consonant interval and a shorter response time to dissonant (less fused) intervals would be consistent with Stumpf's concept. We investigated consonance and dissonance using a timed discrimination task in three experiments.

\section{EXPERIMENT 1}

In Experiment 1, we presented all possible musical intervals to determine which intervals, if any, would produce fusion (a slow response time or a judgment of "one tone" to a presentation of two tones). Since Stumpf's fusion principle has not been tested using methods other than introspection, the purpose of this experiment was simply to try to find nonintrospective evidence that fusion may operate in the perception of musical intervals.

\section{Method}

Subjects. Seven students from an introductory psychology class participated in the experiment for course credit.

Apparatus. A Commodore 64 computer generated and produced the stimuli and collected subjects' responses and response times.

Design. Four blocks of 195 trials were presented. Each contained 65 single tones and 10 each of the following pair types: unison, minor second, major second, minor third, major third, perfect fourth, tritone, perfect fifth, minor sixth, major sixth, minor seventh, major seventh, and octave. These intervals span from 0 to 12 semitones. The unison intervals (zero semitones) were included as a control for the single tones. Although overall intensity on the Commodore 64 is supposed to be equated for composite sounds (whether one, two, or three tones are playing simultaneously), we were concerned that a subtle difference between the one- and two-tone conditions might exist; therefore, we included a two-tone condition with both notes of the same frequency. The frequencies of the tones included the equal-tempered semitone steps between $261.6 \mathrm{~Hz}$ (middle C) and $987.8 \mathrm{~Hz}$ (B5, two octaves above middle C). The specific instances of the intervals presented were chosen randomly from all possibilities within this range. The tones were played using the "square" waveform setting on the Commodore 64, a complex wave sounding similar to an organ tone and described by the manufacturer as "a bright, hollow square wave" (Commodore 64 Programmer's Reference Guide, 1984, p. 464). The tones and tone pairs were arranged randomly within each block.

Procedure. The tones were played through the speaker built into the computer and heard in the open air by the subjects. During a set of practice trials, the subjects were instructed to adjust the intensity of the tones to a comfortable level, and this level was retained throughout the experiment. On each trial, the subject heard a tone or tone pair and then responded by pressing one key (z) on the computer keyboard for a single tone and another key $(/)$ for two simultaneous tones. The tone(s) continued to sound until the subject responded, but the subjects were encouraged to respond as quickly and accurately as they could. The subject then pressed the space bar to hear the next trial. After completing all the trials, the subjects filled out a musical background questionnaire of our own design.

\section{Results and Discussion}

Latency. Single tones as well as unison pairs were excluded from the analyses because the correct responses to them were different from those given to the other intervals, but the unison pairs were very successful as control pairs in that no subject could distinguish between the single tone trials and the unison trials. An initial glance at the response time data revealed no obvious effects of interval type (mean response times in milliseconds were: minor seconds, 521; major seconds, 528; minor thirds, 514 ; major thirds, 511; perfect fourths, 518 ; tritones, 512 ; perfect fifths, 524; minor sixths, 513; major sixths, 515; minor sevenths, 514; major sevenths, 518; octaves, 528). A 4 blocks $\times 12$ intervals $\times 7$ subjects analysis of variance (ANOVA) performed on the mean correct response times revealed no main effect of interval type. There was a significant effect of block $[F(3,18)=28.839$, 


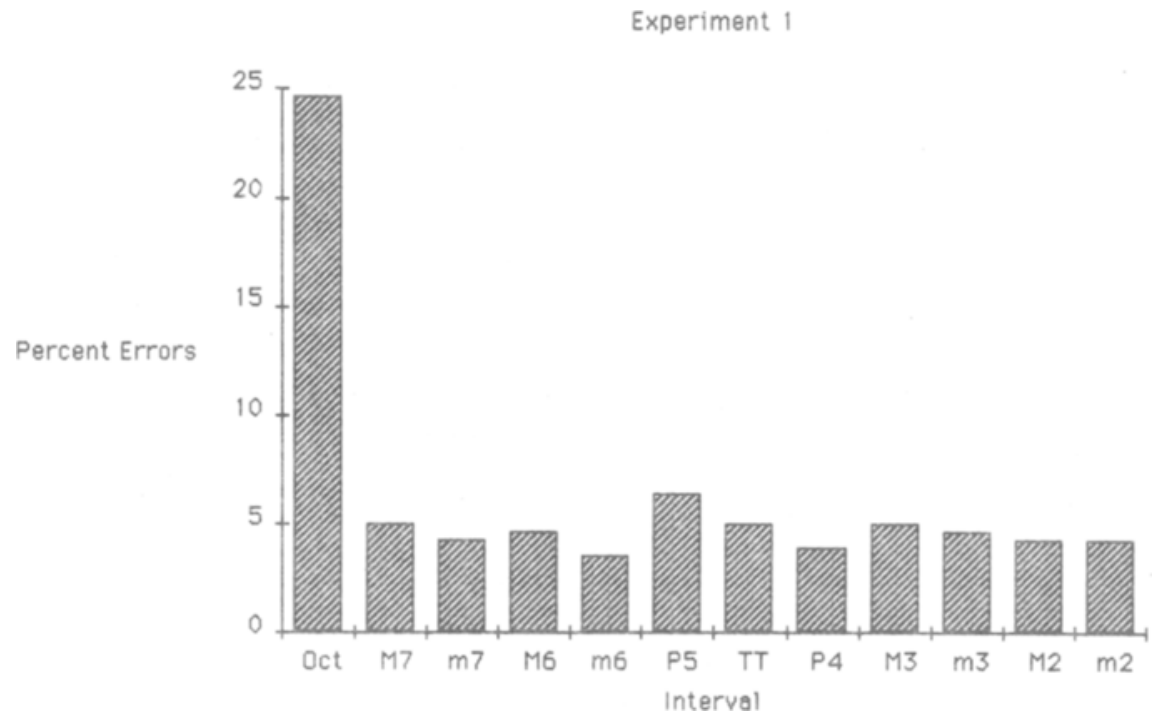

Figure 2. Mean percentage of incorrect ("one tone" to a tone pair) judgments per block as a function of the musical interval of the tone pair (Experiment 1).

$p<.0001]$ due to a decrease in response times across practice (Block 1, 629; Block 2, 496; Block 3, 494; Block 4, 452).

Accuracy. Figure 2 presents the mean percentage of errors per block for all interval types. Obviously, octaves produced more errors than did the other interval types, with perfect fifths a distant second. The analysis of the error data revealed a significant effect of interval type $[F(11,66)=5.128, p<.0001]$. This effect was due to the high error rate for octaves as opposed to the other interval types. No other main effect or interaction was statistically significant.

A comparison of the responses of musicians with those of nonmusicians was not feasible with the small subject sample used in the experiment. However, we were interested to see if only the highly trained musicians were producing more errors to octaves. This was not the case. The three highest error rates for octaves were produced by the most musical subject, the least musical subject, and a third subject with an intermediate amount of training, as identified by our questionnaire.

\section{EXPERIMENT 2}

The large number of errors to octaves in Experiment 1 indicated that fusion might be playing some role in subjects' responses. Resisting a simple dismissal of this result by asserting that "octaves are special" and quitting there, we followed our (and our subjects') intuition that the task in Experiment 1 had been too easy. The second experiment further investigated fusion in interval perception by increasing task difficulty. In the new technique, subjects had to distinguish two-tone combinations from three-tone combinations. Each trial included a "warning tone" an octave below the lower note of the test interval and preceding onset of the test interval by $1,450 \mathrm{msec}$. The ques- tion was thus whether one or two additional tones came after the warning tone had sounded for about $1.5 \mathrm{sec}$. A warning tone an octave below the lower tone in the interval might promote fusion, we thought, by suggesting the harmonic series; the interval of the fifth might then be heard, for example, as the second and third harmonics of the warning tone below.

Additionally, we varied the harmonic richness of the individual tones. Research by Plomp and Levelt (1965) has shown that for intervals made up of pure tones all intervals larger than a "critical band" (approximately a minor third) sound consonant to nonmusicians, whereas intervals falling within this band sound dissonant. However, with intervals made up of more complex tones accompanied by more harmonics, degree of fusion could be influenced by whether or not the harmonics of the two notes coincide or clash. In the case of consonant intervals, such as the perfect fifth, the harmonics of each individual note should coincide with the fundamental and harmonics of the other, enhancing their tendency to fuse. In dissonant intervals, on the other hand, the harmonics of the individual tones do not coincide; rather, they often clash. This tendency of harmonics either to clash or coincide should depend on how many harmonics are actually present in the acoustic signal and on their intensities. With tones approaching sine waves, there will be few or no harmonics either to coincide or to clash with constituents of the other tone. However, with tones accompanied bymore or stronger harmonics, the difference in fusion between consonant and dissonant intervals should be enhanced.

Finally, we varied the tuning systems of the intervals because a "purist" version of the fusion principle would predict that just tuning would produce larger fusion effects than equal-tempered tuning. One of Stumpf's laws of fusion (later modified) was "degree of fusion is a func- 
tion of the vibration ratio of the components" (Boring, 1942 , p. 361). Since intervals tuned in just temperament have simpler frequency ratios than intervals tuned in equal temperament, we believed that they would show a greater tendency to fuse. The difference between temperaments is particularly evident in dissonant intervals such as seconds and sevenths, whereas consonant intervals such as perfect fifths and fourths are only very slightly "mistuned" in equal temperament as compared with just tuning.

A related reason why just-tuned consonant intervals should fuse well involves the harmonic series. Consonant intervals occur relatively early in the harmonic series. The second harmonic is just an octave higher than the fundamental pitch, the third a perfect fifth above the second, and so on. Thus, even when we hear a single musical tone, we actually hear a fused combination of sine-wave components. The lower (and more easily heard) of these components form consonant intervals. Hearing a consonant interval (such as an octave or a perfect fifth) formed by two tones produces an acoustic consequence very similar to that produced by hearing a single harmonically rich tone. A dissonant interval, such as the tritone, however, is not represented early in the harmonic series and therefore does not mimic the tonal consequences of single musical tones. In just temperament, most traditionally consonant musical intervals match exactly the frequencies of sine-wave components resulting from the harmonic series. In equal-tempered tuning, the width of the musical intervals, particularly dissonant intervals, are all slightly 'off' relative to the locations of pitches that would be in exact accord with the harmonic series. Therefore, according to this reasoning, use of just temperament ought to produce stronger fusion effects that should equal temperament, especially for dissonant intervals.

\section{Method}

Subjects. Thirty-six New Haven area summer residents (including both students and nonstudents with a range of musical ability) served as subjects for pay.

Apparatus. A Commodore 64 computer produced the tones, which were fed through a Realistic QA-620 amplifier to a Realistic Solo-103 speaker, and collected response information, as in Experiment 1.

Design. Waveform complexity was a between-subjects factor, and tuning system and interval type were within-subjects factors. One group of 18 subjects heard waveforms with limited harmonic complexity. These waveforms are called "triangle" waves by the Commodore 64 manual (p. 191) and described as "low in harmonics and [having] a mellow flute-like quality"' (p. 464). An analysis of the waveform revealed a rough approximation to a sine wave. The other waveform, designated "square" in the manual, was obviously richer in its harmonics than the simple waveform, both to the listener and according to the corresponding Fourier spectral and waveform diagrams. Although a true triangular wave contains the same harmonics as a square wave-the odd harmonics-the intensity of these harmonics drops off much faster in the triangular wave (as the square of the reciprocal of the number of the harmonic). Thus, the triangular wave results in fewer perceived harmonics and a purer sound.

All subjects experienced two blocks of 180 trials, one block using pitches tuned to just temperament and one block using pitches tuned to equal temperament (see Backus, 1977, pp. 143-148). Each block contained 60 single tones and 20 each of six types of tone pair, three consonant intervals - octave, perfect fifth, and perfect fourth-and three dissonant intervals-major seventh, tritone, and minor second. The frequencies of the tones ranged from 246.93 to $660.00 \mathrm{~Hz}$. Because one of our conditions employed just tuning, in which not all nominally similar intervals have the same frequency rates, two specific instantiations (with the same ratio) of each interval type were chosen from within the frequency range used.

Procedure. As explained above, we attempted to increase task difficulty by adding a "warning tone"' to each trial. Subjects were told that the warning tone would sound and stay on throughout the trial. Shortly $(1,450 \mathrm{msec})$ after the onset of the warning tone, the test item sounded and the subjects responded according to whether they heard one new tone (added to the warning tone) or two new tones. They were instructed to respond as quickly and with as few errors as possible. At the end of the session, the subjects completed the musical background questionniare.

\section{Results and Discussion}

Latency. The shaded bars in Figure 3 show the mean response time for each interval type. As predicted, response times to consonant intervals were longer than response times to dissonant intervals. A 2 waveform $x$ 18 subjects $\times 2$ temperament $\times 6$ interval ANOVA performed on the response times revealed a significant main effect of interval type $[F(5,170)=23.89, p<.0001]$. Newman-Keuls comparisons (as well as contrasts) performed on the means showed that the intervals of the octave and the perfect fifth were responded to significantly more slowly than were the other intervals and also that they differed from each other $(p<.05)$. In addition, reaction times were longer to major sevenths than to minor seconds. No other main effects or interactions were significant.

Accuracy. Figure 4 shows the mean percentage of errors for each interval type. As in Experiment 1, by far the most errors occurred on octave trials, with perfect fifths next. An ANOVA on the error data revealed a significant main effect of interval type $[F(5,170)=33.39$, $p<.001]$. Consistent with the response time data, there were more errors for intervals of the octave and the perfect fifth than for the other intervals and there were significantly more errors for octaves than for fifths (Newman-Keuls comparisons, $p<.05$ ). No other main effects or interactions were significant.

Analysis of "musical" subjects. For the group as a whole, neither tuning system nor waveform contributed reliably to performance. To investigate the possibility that highly trained musicians would be especially sensitive to these factors, the musical background questionnaires were independently ranked according to degree of musical training by two musically trained individuals $(r=.976$, $p<.0001$ ). The data from the 14 most musical subjects were analyzed separately (the median amount of training in this group was several years on at least two instruments in addition to theory training). The unshaded bars in Figure 3 show the mean response times for each interval type for the more musically trained subjects. These data 


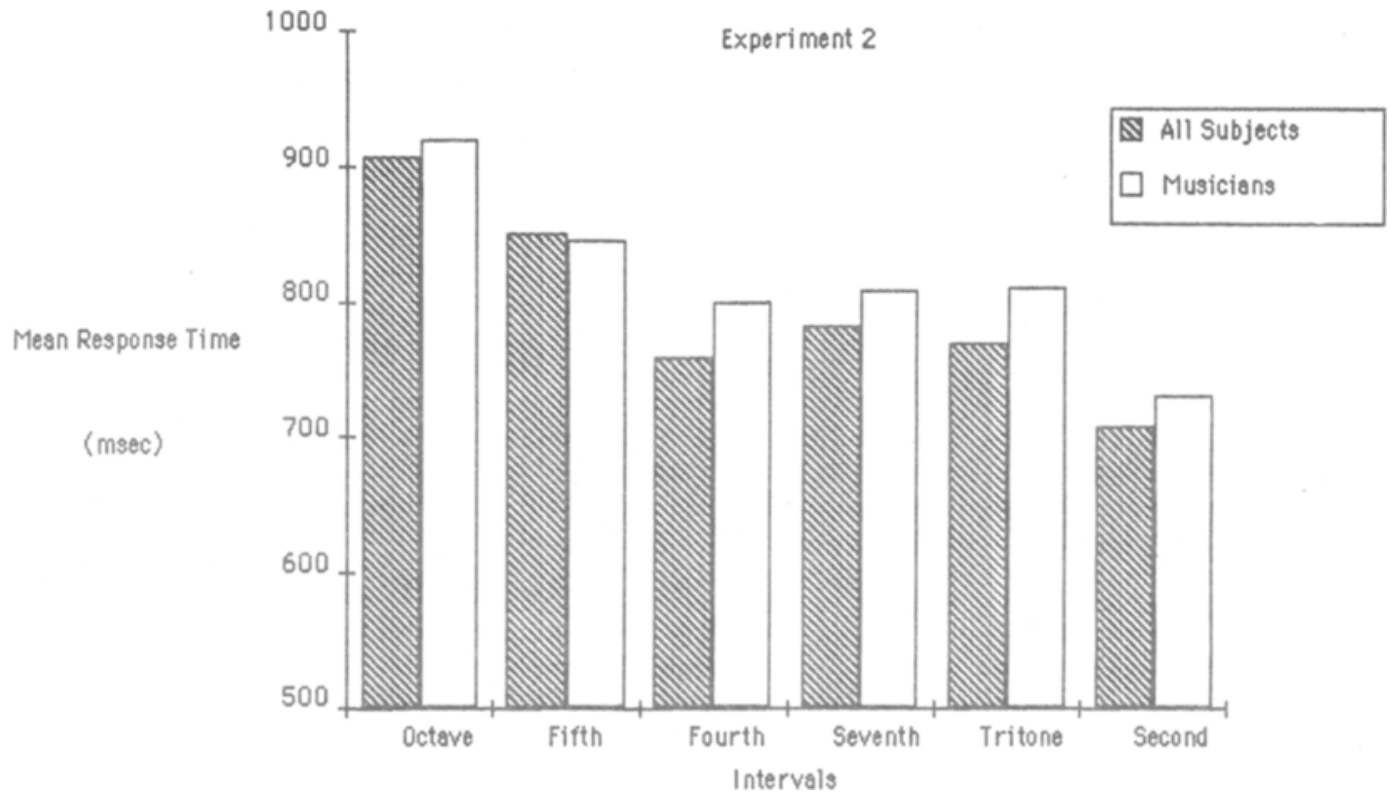

Figure 3. Mean response time (for correct responses) as a function of musical interval (Experiment 2). Data for all subjects $(n=36)$ are represented by the shaded bars. Data for the 14 most musical subjects are represented by the lighter bars.

show the same effect as the data on the whole, a main effect of interval type with consonant intervals producing longer response times than did dissonant intervals $[F(5,60)=5.54, p<.01]$. No other main effects or interactions were significant.

Figure 5 shows the mean percentage of errors for each interval type in both tuning systems for the same 14 more musical subjects. Once again, consonant intervals produced more errors than did dissonant intervals $[F(5,60)=12.27, p<.001]$. In addition to the main effect of interval, which was significant for the group as a whole, the interaction of temperament and interval type, not significant for the group, was significant for the trained musicians $[F(5,60)=2.85, p<.05]$. This was due to the musicians' making more errors to consonant intervals in just tuning than to consonant intervals in equal temperament. Perhaps, as we conjectured above, just-tuned intervals promote fusion because the harmonics of the two tones are more closely aligned than they are in equal temperament.

The interaction between interval and temperament (Figure 5) is not without ambiguity, however. In the first

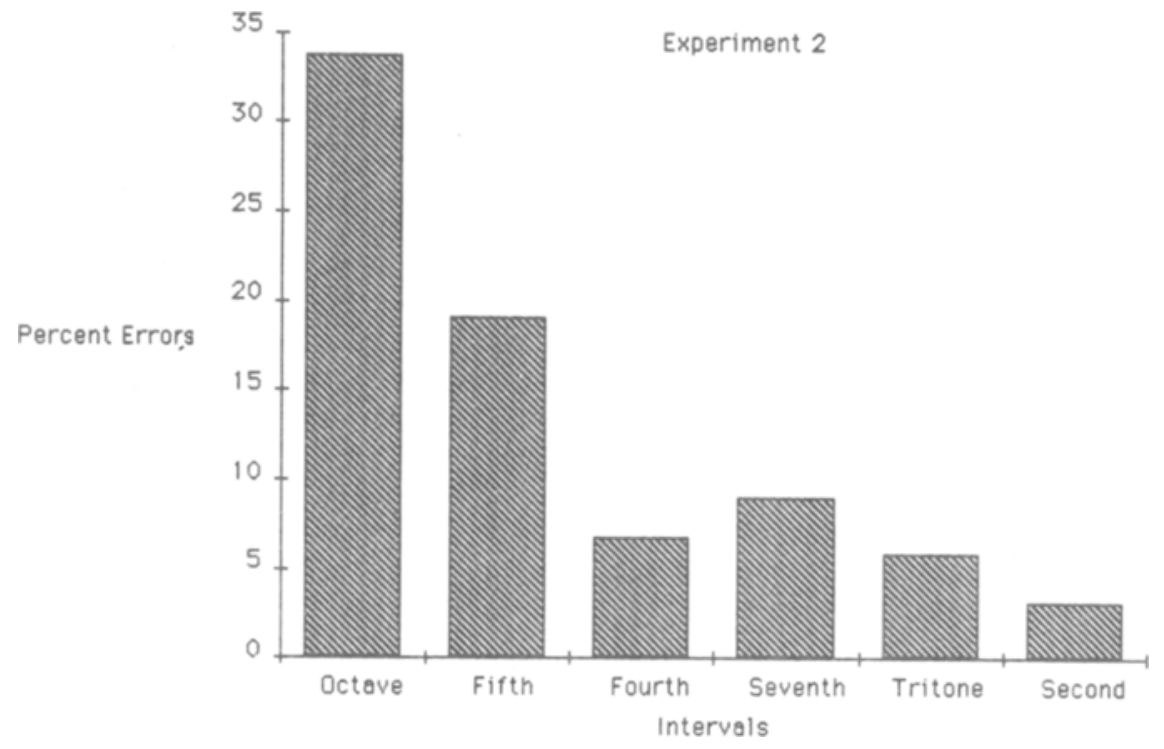

Figure 4. Mean percentage of incorrect ("one tone" to a tone pair) judgments as a function of the musical interval of the tone pair (Experiment 2). 


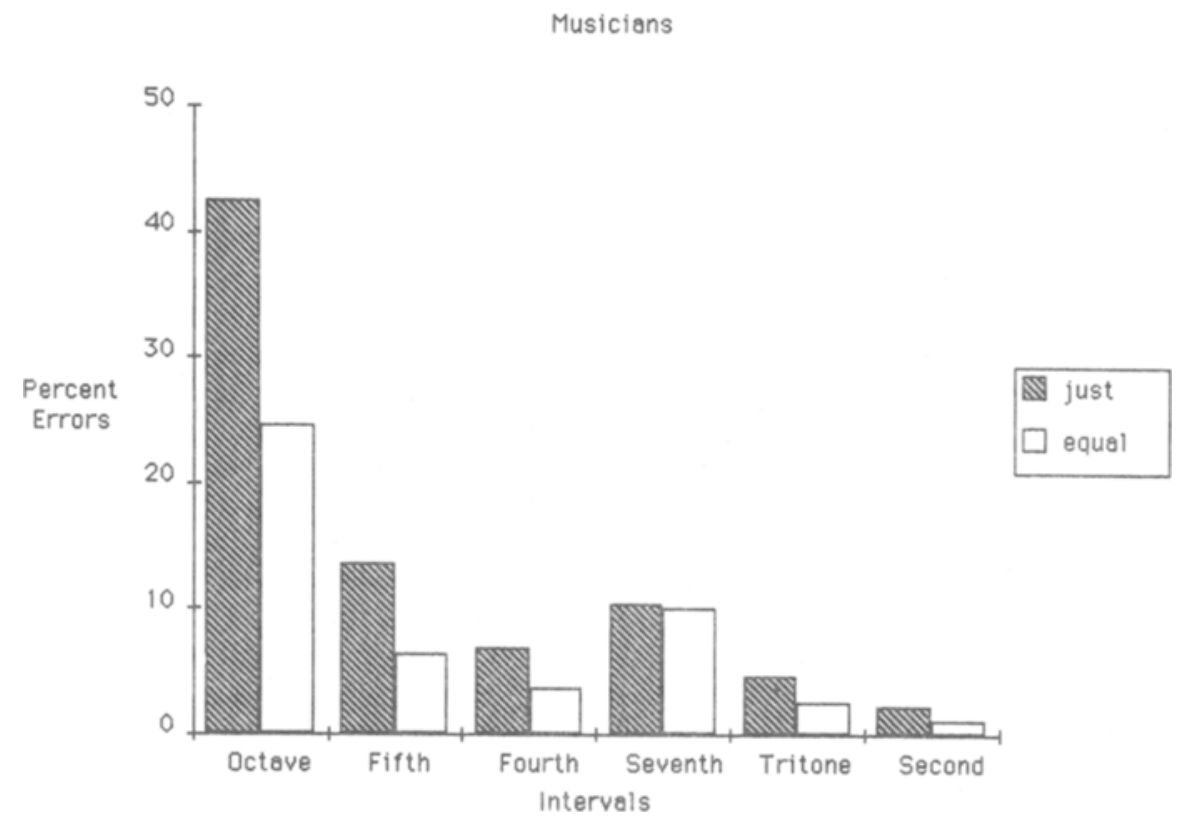

Figure 5. Mean percentage of incorrect ("one tone" to a tone pair) judgments by the 14 most musical subjects as a function of the musical interval of the tone pair for both just- and equaltempered tuning (Experiment 2).

place, Stumpf himself believed that these subtle tuning differences would not generally affect judgments of fusion (Boring, 1942). Moreover, since the differences between just and equal temperament, in terms of simple integer ratios and alignment of harmonics, occur mainly in the dissonant intervals, the difference in errors between just and equal temperament among consonant intervals is paradoxical. Specifically, of the intervals used in Experiment 2, although the major seventh and the minor second differ the most from just to equal temperament (11.7 cents in each case; see Burns \& Ward, 1982), these two intervals did not show the greatest difference in errors between temperaments. On the other hand, octaves do not differ at all from just to equal temperament and perfect fifths and fourths differ only slightly ( 2 cents), yet they produced the greatest difference in the number of errors. We find it tantalizing that the trained musicians (who would be expected to have "good ears") were the ones to show a difference due to tuning system, but we do not yet understand the result in detail. However, the data are broadly consistent with the work of Plomp and Levelt (1965), who found sharp peaks of perceived consonance around simple integer ratio intervals. Thus, a sensitivity to mistuning of consonant intervals but not to dissonant intervals could be rationalized.

In summary, our complication of the task in Experiment 2 was successful in producing a more informative pattern of results than that of Experiment 1 . In the second study, we were able to detect an influence of interval on reaction times as well as on accuracy. Moreover, the fusion obtained was not limited to the octave condition. Our completely straightforward expectations for waveform complexity and tuning system manipulations were largely unconfirmed in this study, except that the more musical subjects showed more fusion with just-tuned consonant intervals than with the other intervals. It is possible that the two waveforms used in Experiment 2 were not different enough to influence fusion, even though they sounded very different in tone quality, an impression that was confirmed by inspection of the waveforms.

\section{EXPERIMENT 3}

In Experiment 2, we added a warning tone to make the decision more complex. This warning tone was an octave below the lower tone of the test interval. This may have produced fusion based on the harmonic series. The intervals occurring lower in the harmonic series are also the ones traditionally considered consonant. This line of reasoning suggests that the auditory system has some tendency to organize as single events pitches that could have resulted from the harmonic series. This would result in observed fusion, which in turn might produce the "pleasant" subjective response. To determine whether the fusion found in Experiment 2 occurred in this way, Experiment 3 measured fusion using warning tones that were not all in octave relationship to the lower tone of the test interval. Three types of warning-tone interval were used, an octave, a perfect fifth, and a tritone below the lower tone of the test interval. If the warning tone in Experiment 2 served to suggest the harmonic series and caused the test interval to fuse, then nonoctave warning tones, particularly the tritone, should result in less fusion.

To better test the harmonic-series explanation for fusion, we changed, in Experiment 3, some of the six interval types used in Experiment 2. The octave, perfect 
fifth, and perfect fourth were retained as consonant intervals. These are the first three intervals formed by adjacent components of the harmonic series. Major thirds, minor thirds, and major seconds (the next three intervals formed by adjacent members of the harmonic series) were substituted for the tritone, major seventh, and minor second as dissonant intervals. Since the thirds are technically considered "imperfect consonances,"' these six intervals represent a continuum from most consonant (octave) to least consonant (major second) better than do two distinct (consonant and dissonant) groups. Inclusion of thirds could also result in a better test of differences in amount of fusion between intervals in just and equal temperament, since the intervals of the major and minor third contain a greater degree of deviation between the two temperaments than do the minor second, tritone, and major seventh used in Experiment 2.

\section{Method}

Subjects. Twenty Yale introductory psychology students participated in the experiment for course credit.

Apparatus. The apparatus was the same as that used in Experiment 2.

Design. The blocks of 216 trials were presented to the subjects. One block used intervals tuned in just temperament; the other block used equal temperament. The order of presentation of the two blocks was counterbalanced. Each block contained 54 unison trials and 27 each of the six types of tone pair-octave, perfect fifth, perfect fourth, major third, minor third, and major second. The three types of warning-tone interval-octave, perfect fifth, and tritone-were evenly paired with all the trial types, and the trials were ordered randomly.

\section{Results and Discussion}

Latency. Overall, the manipulation of warning-tone interval influenced response times exactly as we had expected. Response times were longest for the octave con- dition ( $841 \mathrm{msec})$, intermediate for the perfect-fifth condition $(800 \mathrm{msec})$, and shortest for the tritone condition $(772 \mathrm{msec})$. A 20 subjects $\times 2$ temperament $\times 3$ warning tone $\times 6$ interval ANOVA performed on the response time data indicated that this main effect was highly reliable $[F(2,38)=15.87, p<.0001]$. NewmanKeuls comparisons $(p<.05)$ of the three warning-tone conditions showed that each differed reliably from the other two.

The overall pattern of response times for the six types of test interval was consistent with that found in Experiment 2 . Response times increased when the intervals were more consonant. In other words, it took subjects longer to respond "two tones" to more consonant intervals than to more dissonant intervals. This effect of interval type was significant $[F(5,95)=11.97, p<.0001]$ and, by ignoring the warning-tone breakdown, can be seen in Figure 6. Newman-Keuls comparisons of the means showed that octaves were responded to more slowly than were all the other interval types and that fifths were responded to more slowly than were minor thirds and major seconds.

The profile of response times across intervals was dependent on the preceding warning tone, as shown in Figure 6. The octave warning tone not only resulted in longer response times overall but also in longer response times for the consonant intervals of the octave and perfect fifth than for the other intervals. We are tempted to attach some importance to the octave warningtone-perfect-fifth interval arrangement because it mimics exactly what one hears when the fundamental and the second and third harmonics of a complex tone are sounded. The other two warning tones did not differ very much in their effect on response time for different intervals, although the perfect-fifth warning tone resulted in longer response times to the octave interval than did the tritone

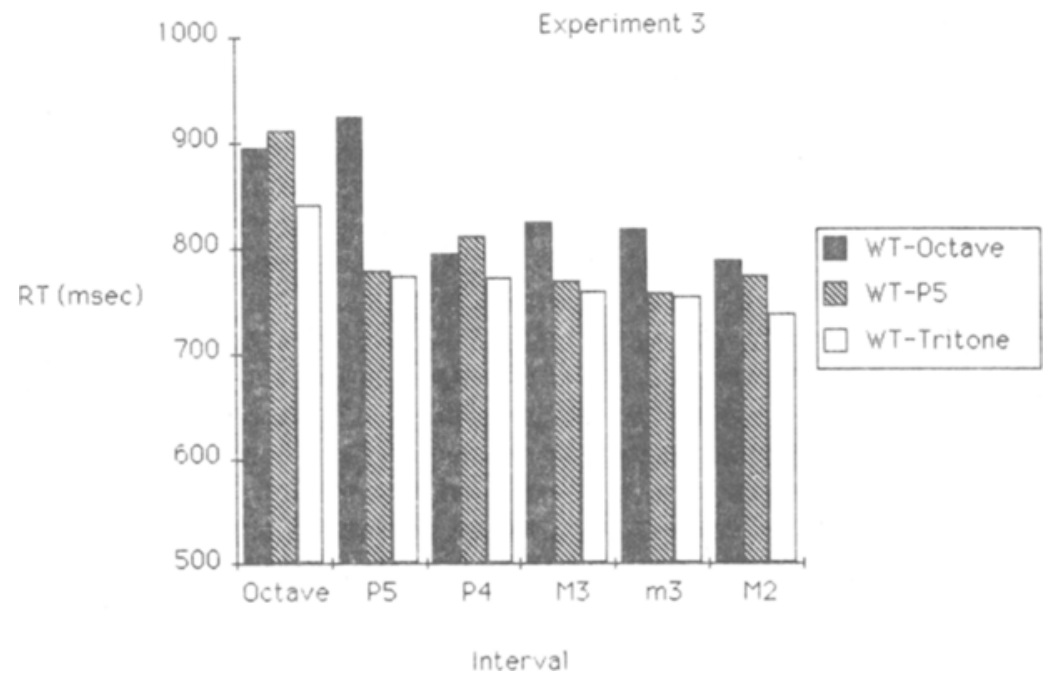

Figure 6. Mean response time as a function of interval for three warning-tone conditions, octave, fifth, and tritone (Experiment 3). 
warning tone. This interaction of warning tone and interval type was significant $[F(10,190)=2.43, p<.01]$.

The effect of interval also depended on temperament $[F(5,95)=2.42, p<.05]$. As shown in Figure 7, octave intervals in the just-temperament condition were responded to more slowly than were octaves in the equaltemperament condition. This same difference was found for the musical subjects of Experiment 2 (Figure 5). As explained above, this result is somewhat baffling, since the octave intervals heard in the two conditions are acoustically identical. One can only posit some kind of contextual explanation for the difference between the two conditions. For example, the equal-temperment condition, in which all the intervals except the octave are slightly mistuned (relative to the simple integer properties of the harmonic series), might cause subjects to shift their fusion criteria, demanding less evidence before correctly responding "two tones" than they would in the justtemperament conditon. This may explain why intervals identical in the two conditions would produce such different patterns of responses.

Accuracy. Analysis of the error data revealed effects consistent with the response time data. The mean percentage of errors for the octave, perfect-fifth, and tritone warning-tone conditions were 20,14 , and 11.8 , respectively $[F(2,38)=25.08, p<.0001]$. The three conditions all differ significantly from one another (NewmanKeuls comparisons, $p<.05$ ).

The overall effect of interval type can be seen in Figure 8 by ignoring the warning-tone breakdown. As in Experiments 1 and 2, octave intervals resulted in the greatest number of errors, followed by perfect fifths and perfect fourths, with the less consonant intervals receiving more correct judgments. This reliable main effect of interval type $[F(5,95)=14.50, p<.0001]$ was further explored with Newman-Keuls comparisons $(p<.05)$, establishing reliable differences between the octave and all other intervals as well as between the fifth and the major second. The interval effect interacted with the warning-tone condition, as shown in Figure 8 $[F(10,190)=9.20, p<.0001]$. As in Experiment 2, the octave warning-tone condition resulted in more errors to intervals of an octave or a perfect fifth. As explained above, the octave warning-tone-perfect-fifth combination replicates, in order, the first three components of the harmonic series. In addition, the effect of the perfect-fifth warning tone on the interval of a perfect fourth is of particular interest to a discussion of a harmonic series explanation of fusion. The perfect-fifth warning tone resulted in a greater number of errors in the perfect-fourth interval condition than did either of the other two warning tones. Although the octave as warning tone generally led to more errors than did the fifth, this result was reversed for the perfect-fifth-perfect-fourth combination. This combination could be heard as the second, third, and fourth harmonics of a missing fundamental below, resulting in greater (con)fusion in deciding if one or two tones (in addition to the warning tone) were being heard. Figures $9 \mathrm{a}$ and $9 \mathrm{~b}$, moreover, show that this special difficulty with the perfect fourths heard against the background of a perfect-fifth warning tone was more apparent in the just-temperament condition (Figure 9a) than with intervals of equal temperament. Because notes tuned in the just temperament line up precisely with the elements of the harmonic series, it was predicted that more fusion would be evidenced in this condition than in equal temperament, particularly with the octave and perfect-fifth warning tones preceding consonant intervals. Indeed, the temperament $x$ warning tone $X$ interval interaction was statistically reliable $[F(10,190)=2.48, p<.01$; see

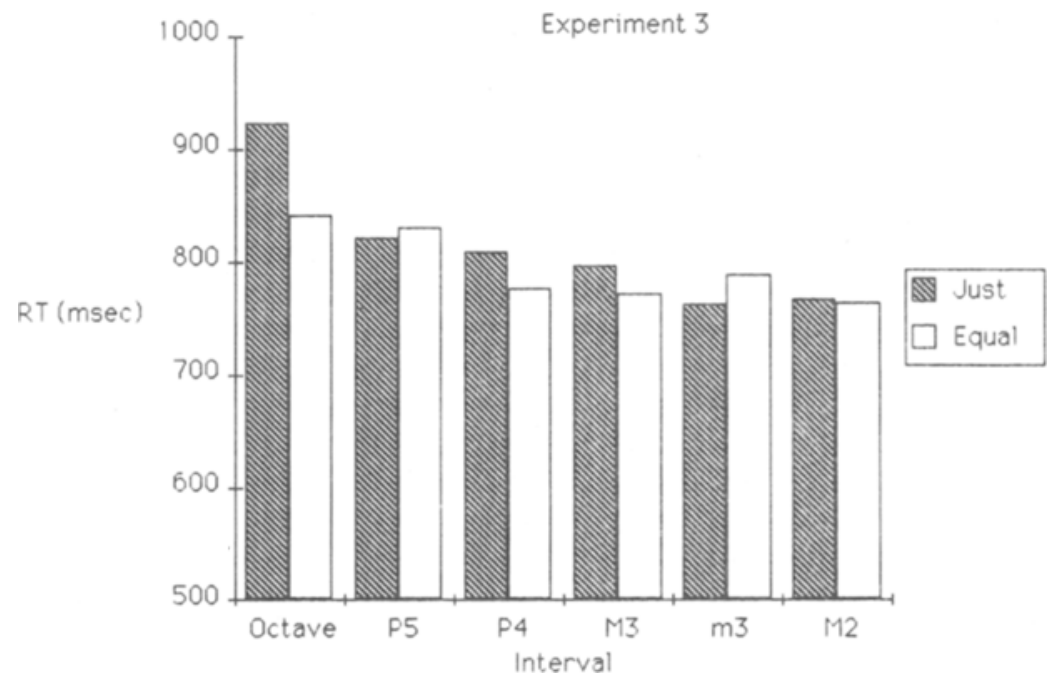

Figure 7. Mean response time as a function of interval for two temperament conditions, just and equal (Experiment 3). 


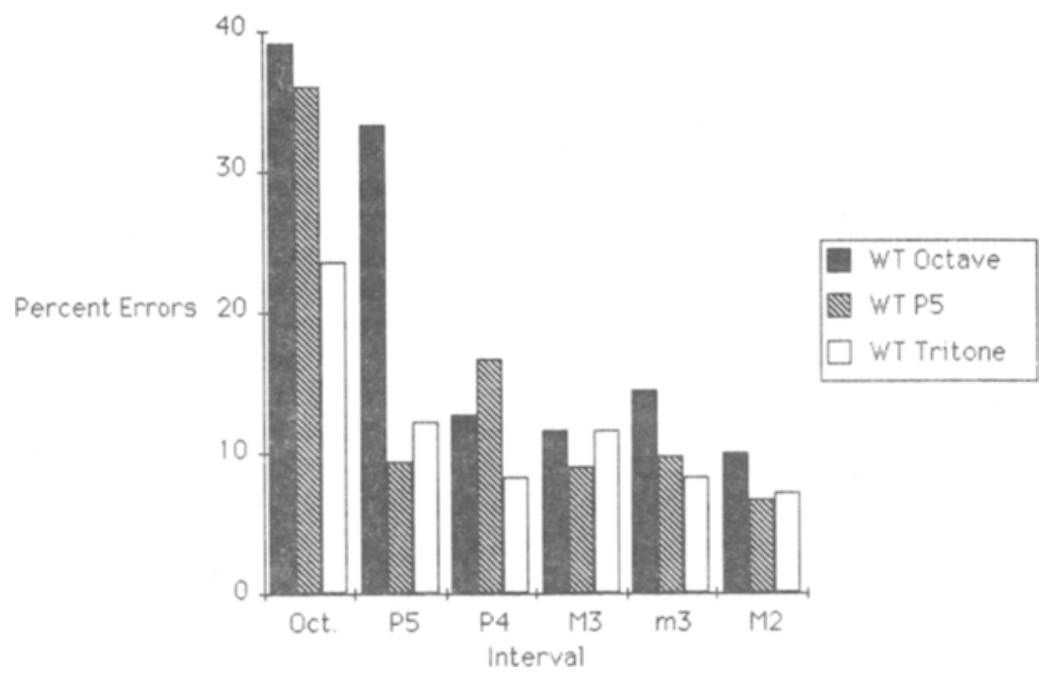

Figure 8. Mean percentage of errors ("one tone added" to tone pair added condition) as a function of interval type for the three warning-tone conditions, octave, fith, and tritone (Experiment 3).

Figures 9a and 9b] evidently owing largely to this predicted effect.

Analysis of "musical" subjects. To investigate the possibility that more musical subjects might be more (or less) inclined to hear warning-tone-plus-interval combinations as fused harmonic components as opposed to chords, the musical background questionnaires were independently rank-ordered by two trained musicians $(r=.86$, $p<.001$ ). A median split of the averaged ranks produced two equal-sized groups. (The level of training of the subjects in Experiment 3 was generally lower than that of the subjects in Experiment 2. No subject in Experiment 3 had had any theory training, and few had had training on more than one instrument.) An ANOVA performed on the response time data including musical/nonmusical groups as a factor revealed only a main effect of group. Musicians responded more quickly overall than did nonmusicians [681 vs. $928 \mathrm{msec}$, respectively; $F(1,18)=$ $5.79, p<.028]$. No interactions of other factors with musical group were significant.

The analysis of the error data by musical group revealed no main effect of group. The only interaction with group that reached significance was a group $\times$ temperament $\times$ interval interaction $[F(1,18)=3.178, p<.01]$. This interaction, shown in Figure 10, is the result of musicians' making more errors to just-tuned fifths, fourths, and major thirds than to the intervals in equal temperament and than the nonmusicians did to those intervals in either temperament. This result would be expected if the musicians were more sensitive to slight acoustic differences such as are caused by temperament. The result is also consistent with the findings for musicians in Experiment 2, even though the level of musical training was lower in Experiment 3.
The analysis by musical group revealed nothing to contradict any of the overall findings.

Thus, the additional manipulations of Experiment 3 have supported the harmonic-series explanation of fusion suggested by the results of Experiment 2. Intervals that could be heard as consisting of the harmonics of a present (or missing) fundamental were more likely to fuse, resulting in either errors or a longer time to correctly decide that more than one tone had been heard. On the other hand, intervals not consistent with the harmonic series were less inclined to fuse.

Our emphasis on the harmonic series as promoting fusion of tones in our experimental situation should not be taken as an article of blind faith that spectral processes are the cause of fusion. Bregman, Abramson, Doehring, and Darwin (1985) have pitted spectral factors against periodicity factors in an experiment on the fusion of sinewave components. With amplitude-modulated signals, the spectral manipulation was whether or not the individual sinusoids in complex tones could have been harmonics resulting from a fundamental pitch and the periodicity manipulation was the modulation frequency of the components. In their study, complex tones fused on the basis of common modulation frequencies, even if the partials were not harmonically matched; spectral factors, pitch and harmonics, were less important. Because we varied only spectral factors here, we obviously cannot comment on the issues studied by Bregman et al. (1985).

\section{GENERAL DISCUSSION}

We began this inquiry on the consonance/dissonance problem by mentioning the opposition of two viewpoints. 

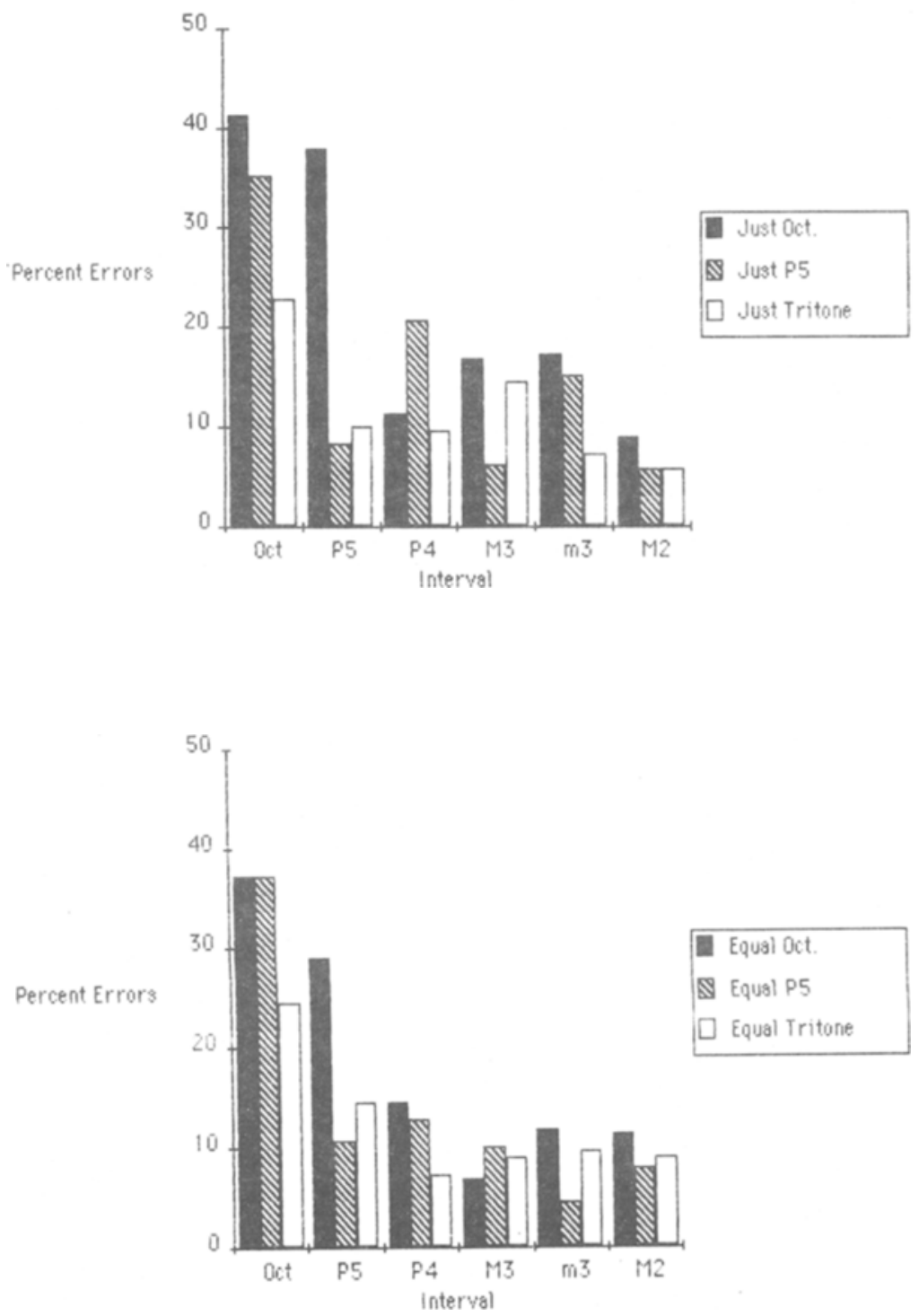

Figure 9. Mean percentage of errors as a function of interval for the three warningtone conditions in just temperament (a) and equal temperament (b).

The rationalist tradition, with a genealogy reaching from Pythagoras to Helmholtz, takes inspiration, in one way or another, from the properties of the harmonic series. The empiricist or "brainwashing" tradition, a more recent attitude, is solidly committed to a general environmentalist approach.

Both sides of the question have their strong and weak points: Rationalists note that the hierarchy of conventionally consonant intervals-from octave down-follows with great (though not complete) precision the series of successive intervals realized in the harmonic series. Radical empiricists must swallow hard and maintain that this correlation is a plain coincidence. On the other hand, the harmonic series fails notoriously to provide a rational basis for the diatonic scale. The strong point of the empiricist argument is historical change in what tone combinations people consider consonant and dissonant. Slominsky (1965) has documented, with reprints of 150 years of concert reviews, that what may sound bland today struck some listeners as ear-splitting noise not long ago. Furthermore, the relative acceptability of certain musical intervals, notably the perfect fourth, has changed within historical spans for which we have written records. On the other hand, rationalists would respond, the consonant 


\section{Experiment 3}
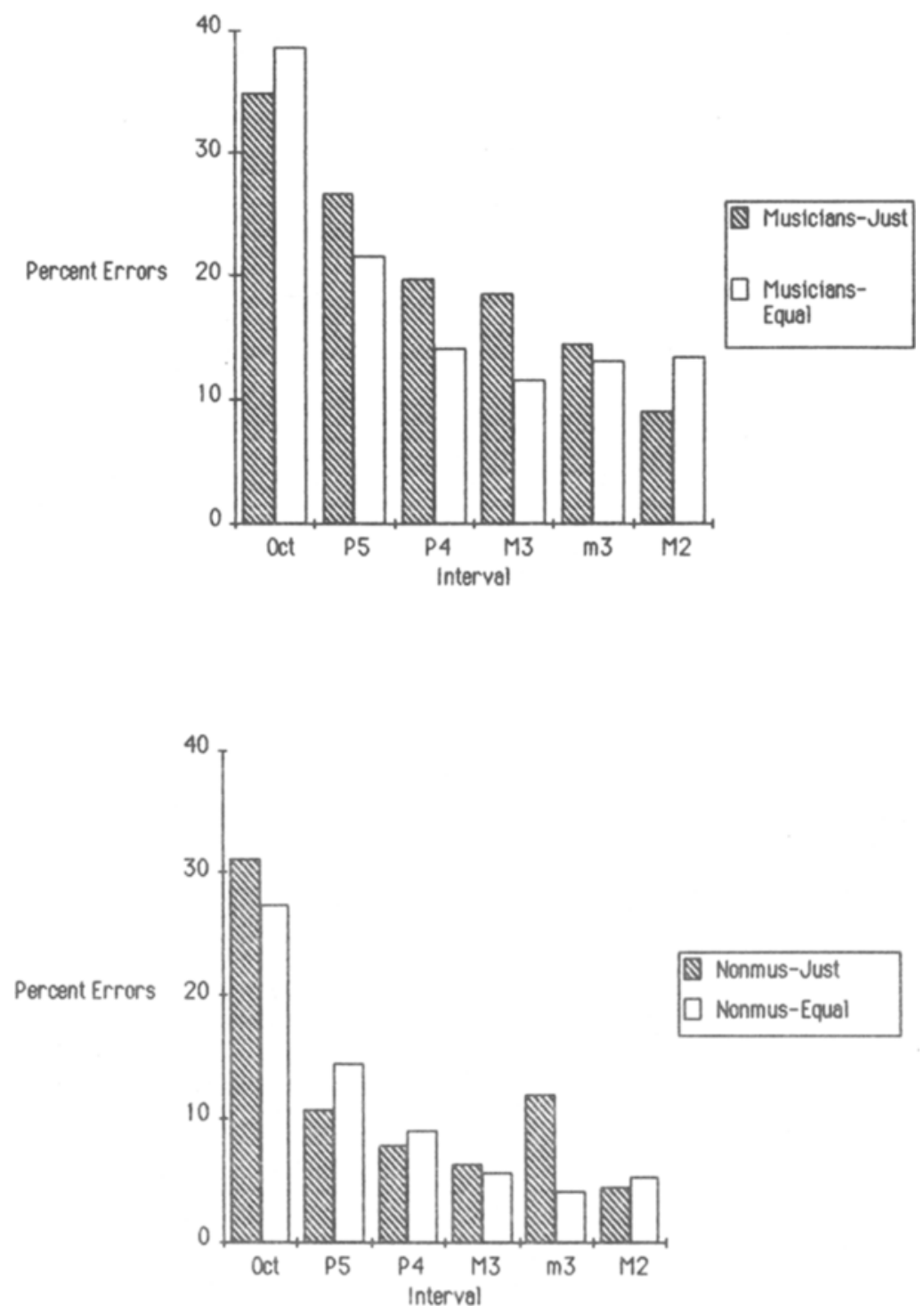

Figure 10. Mean percentage of errors for musicians and nonmusicians as a function of interval for just and equal temperament.

status of the earliest intervals in the series, for example the octave, has not been challenged and may even be universal across musical cultures (Dowling \& Harwood, 1986, chap. 4).

Part of the difficulty has been resolved, as we said above, by distinguishing musical from tonal consonance and dissonance. Although this distinction has allowed investigators, including us, to restrict themselves to the more tractable problems facing the interpretation of tonal consonance/dissonance, it only postpones theoretical work on the most basic question, which asks how tonal and musical consonance are related to one another. Stated this way, the rationalist and empiricist viewpoints are not as different from one another as is generally thought. For example, one can derive the correlation between traditional consonance judgments and the harmonic series on empiricist principles: The argument begins with the incontestable fact that every time we hear a musical sound, we are necessarily hearing some number of harmonics marching up the frequency scale as even-number multiples of the fundamental pitch and gradually weakening in intensity. Add to this fact the assumption that familiarity with any stimulus engenders positive affect (Zajonc, 1980) and it follows that octaves should be the most preferred intervals and those intervals absent from the harmonic series or only weakly represented, the least preferred. Thus, an interpretation based on experience can be fully consistent with the acoustical properties of musical tones. 
Perhaps, then, we shall have to resort to studies of infants (Demany \& Armand, 1984) to settle the role of experience in the matter.

Our three experiments are broadly consistent with an attitude that continues from the assumption that the harmonic series is central to tonal fusion. In all three experiments, combinations of pitches that could be interpreted as deriving from harmonics based on some fundamental frequency were harder or slower to classify as multiple tones than those that could not be interpreted that way. For the reasons just explained, we cannot use this evidence to make claims about the ultimate source of tonal consonance and dissonance. What, then, do we see as the progress represented by our findings on the fusion of musical intervals?

First and most important, our experiments furnish a new and converging experimental realization of a concept whose behavioral measurement has long depended only on subjective intuitions. As long as intuitive qualities are consensual, they are not inherently ineligible as scientific concepts, as research on visual imagery and even color perception has shown. However, the pleasantness of consonant intervals is contaminated to an unknown degree by prior expectations and experimental demand characteristics. The speed and accuracy of responses in the fusion task used here are certainly less contaminated in this way, and their convergence with verbal ratings should therefore be taken seriously.

In another effort to provide convergence of this sort, van deGeer, Levelt, and Plomp (1962) used a series of semantic scales (including "sounds like one tone-sounds like more tones," "smooth-rough," and "consonant-dissonant") to evaluate "the connotation of musical consonance." They found consonance to be connotatively quite different from fusion. Perhaps our measures, response time and errors, tap the perception of fusion more directly and sensitively than does semantic scaling.

A second potential use of the fusion task might be to construct a scale of perceived tonal consonance. Although strong rational arguments-both acoustical and musictheoretical-for the particulars of such a scale date back at least to Helmholtz $(1877 / 1954)$, the evidence for the relative consonance of different intervals is not abundant. Specifically, Kameoka and Kuriyagawa (1969) offered data confirming the predicted sharp gradients of consonance for intervals surrounding the simple integer frequency ratios; however, they did not address the question of the relative consonance of the intervals occurring at the simple integer "peaks." The fusion task might be useful in defining a scale of consonance/dissonance. We ourselves are not commited to this goal. For one thing, our experiments did not reveal reliable differences beyond the perfect fifth and perhaps the perfect fourth. Although larger experimental efforts might bring out such differences, say, establishing slower times or more errors for thirds, the cost in effort might be exaggerated.

Finally, our data make a case for associating the consonance of musical intervals with detailed properties of the harmonic series. The third experiment was especially provocative in this way, showing as it did that extra response time and errors resulted when the entire configuration of background warning tone and stimulus interval could have resulted from a single musical tone with harmonics.

Whatever solutions lie ahead for the problems we raise here, we may now finally elevate Stumpf's principle of fusion from conjecture to established fact.

\section{REFERENCES}

APEL, W. (1972). The Harvard dictionary of music (2nd ed.). Cambridge, MA: Belknap Press of Harvard University Press.

Backus, J. (1977). The acoustical foundations of music. New York: Norton.

BorING, E. G. (1942). Sensation and perception in the history of experimental psychology. New York: Appleton-Century Crofts.

Bregman, A. S., Abramson, J., Doehring, P., Darwin, C. J. (1985). Spectral integration based on common amplitude modulation. Perception \& Psychophysics, 37, 483-493.

BURNS, E. M., \& WARD, W. D. (1982). Intervals, scales, and tuning. In D. Deutsch (Ed.), The psychology of music. New York: Academic Press.

CAzDEN, N. (1980). The definition of consonance and dissonance. International Review of the Aesthetics and Sociology of Music, 2, 123-168.

Commodore 64 Programmer's Reference Guide. (1984). Wayne, PA: Commodore Business Machines, Inc.

Demany, L., a ARmand, F. (1984). The perceptual reality of tone chroma in early infancy. Joumal of the Acoustical Society of America, 76, 57-66.

Dowling, W. J., Hakwood, D. L. (1985). Music cognition. New York: Academic Press.

HeLmholtz, H. L. F. voN (1954). On the sensation of tone: As a physiological basis for the theory of music. New York: Dover. (Original work published 1877)

Kameoka, A., \&uriyagawa, M. (1969). Consonance theory: Part 2. Consonance of complex tones and its calculation method. Journal of the Acoustical Society of America, 45, 1460-1469.

Plomp, R., Levelt, W. J. M. (1965). Tonal consonance and critical bandwidth. Joumal of the Acoustical Society of America, 38, 548-560.

RoberTs, L. A. (1983). Consonance and dissonance: A review of the literature. Unpublished manuscript.

SLominsky, N. (1965). Lexicon of musical invective. Seattle: University of Washington Press.

TerhardT, E. (1984). The concept of musical consonance: A link between music and psychoacoustics. Music Perception, 1, 276-295.

van deGeer, J. P., Levelt, W. J. M., \& Plomp, R. (1962). The connotation of musical consonance. Acta Psychologica, 20, 308-319.

Znonc, R. B. (1980). Feeling and thinking: Preferences need no inferences. American Psychologist, 35, 151-175.

(Manuscript received February 10, 1986; revision accepted for publication October 12, 1986.) 